\title{
Serum mannan-binding lectin in patients with pulmonary tuberculosis: Its lack of a relationship to the disease and response to treatment
}

\author{
Fatemeh Ahmadi ${ }^{1}$, Ataallah Ghadiri ${ }^{2}$, Roohangiz Nashibi², Fatemeh Roozbeh ${ }^{2 *}$, Reza Alizadeh-Navaei ${ }^{3}$
}

Received: 31 Aug 2016

Published: 30 Sep 2017

\section{Abstract}

Background: Lectin pathway mediates complement activation, which is activated by many microorganisms. This study aimed at determining the serum levels of mannose-binding lectin (MBL) in patients with pulmonary tuberculosis, assessing its relationship to antiuberculosis treatment response, and comparing them with a control group.

Methods: This cross-sectional study was conducted on patients with pulmonary tuberculosis during 2012 and 2013 in South West of Iran. PPD-ST-negative individuals were selected as controls from healthy relatives of patients. Serum MBL levels were measured using ELISA kit (Human MBL HK323, Hycultbiotech Company, Netherlands). All patients were followed- up for response to treatment. We applied Mann-Whitney and Fisher's exact tests and used SPSS Version 17 software for statistical analysis.

Results: The study included 62 patients as the case group and 63 noninfected TB patients as the control group. The MBL (ng/mL) in patients with pulmonary tuberculosis (median $=1012)$ was significantly $(\mathrm{p}=0.037)$ higher than that of the control group (median= 296.2). No significant difference was found in the MBL level $(\mathrm{ng} / \mathrm{mL})$ between patients with response to antituberculosis treatment $($ median= 1012) and patients with treatment failure (median= 798.9) $(\mathrm{p}=0.84)$.

Conclusion: MBL may be involved in the pathogenesis of tuberculosis and in the low values that are protective against tuberculosis, and it seems that it has no effect on the antituberculosis treatment response.

Keywords: Tuberculosis, Mannose Binding Lectin, Immune System

Copyright $\odot$ Iran University of Medical Sciences

Cite this article as: Ahmadi F, Ghadiri A, Nashibi R, Roozbeh F, Alizadeh-Navaei R. Serum mannan-binding lectin in patients with pulmonary tuberculosis: Its lack of a relationship to the disease and response to treatment. Med J Islam Repub Iran. 2017 (30 Sep);31:66. https://doi.org/10.14196/mjiri.31.66

\section{Introduction}

About a third of the world population is exposed to Mycobacterium tuberculosis infection (1), which result in 3.1 million annual deaths worldwide (2). However, only $10 \%$ of the infected individuals with $M$. tuberculosis have the active form of the disease. Long-term therapy, drug resistance, the absence of an effective vaccine, and incomplete understanding of the factors controlling the progression of the disease or infection are barriers to control the disease (3); susceptibility to tuberculosis is multifactorial. The active form of the disease resulting in the interaction between the host and pathogen is influenced by genetic and environmental factors, and the host's numerous genes are involved in this process (4-6). Twin studies revealed that the host's genetic factors play an important role in determining susceptibility to infection with Mycobacte-

Corresponding author: Dr Fatemeh Roozbeh, fatemehroozbeh@gmail.com

1. Health Research Institute, Infectious and Tropical Diseases Research Center, Ahvaz Jundishapur University of Medical Sciences, Ahvaz, Iran.

2. Immunology Department, School of Medicine, Ahvaz Jundishapur University of Medical Sciences, Ahvaz, Iran.

3. Gastrointestinal Cancer Research Center, Mazandaran University of Medical Sciences, Sari, Iran. rium tuberculosis and the clinical progression of the disease (7). The immune system defends the first place to deal with this disease, in which the complement system is an important component of innate immunity. Complement is activated through 3 paths to reach the $\mathrm{C} 3$ level and to have a complex membrane attack formation. These pathways include the classical pathway, the alternative pathway, and the mannose-binding lectin (MBL) pathway (8). Lectin pathway is activated by activation of MBL or Ficolin that can recognize carbohydrate ligands of many microorganisms (9). MBL has 6 subunits and is similar to $\mathrm{C} 1 \mathrm{q}$ in structure. Plasma levels of MBL can vary up to 1000 times (10). Genetic differences could influence the MBL level $(11,12)$. In HIV patients, individuals with MBL deficiency progress to AIDS more rapidly. Similar-

$\uparrow$ What is "already known" in this topic:

MBL have a role in immunity system and changes in the serum levels of MBL could result in the onset of TB or its development.

$\rightarrow$ What this article adds:

The low values of MBL are protective against tuberculosis and it has no effect on the antituberculosis treatment response. 
ly, in several studies, MBL deficiency or MBL2 gene polymorphism were shown to have an important role in the development of tuberculosis and meningitis in HIV patients (13). However, it seems that the role of MBL in mycobacterium is not clear and studies on the role of MBL in TB are controversial $(14,15)$.

MBL have shown to have a role in immunity system; moreover, genetic differences of MBL could influence its serum levels in different people. Therefore, changes in the serum levels of MBL could result in the onset of TB or its development. Given the role that genetics play in TB, this study aimed at determining the serum levels of MBL in patients with pulmonary tuberculosis compared to a control group.

\section{Methods}

This cross-sectional study was conducted on 62 patients with pulmonary TB and 63 noninfected individuals in South West of Iran during 2013 and 2014. The inclusion criteria were as follow: adult patients (older than 18 years) with pulmonary tuberculosis in Ahwaz, who were diagnosed and treated with antituberculosis. Exclusion criteria were HIV, cancer, any serious disease, and metabolic and autoimmune disease. Diagnosis of TB was made based on clinical symptoms, radiological and microbiological criteria (smear positive) published in the national protocol by infectious disease specialists. The non-TB control group (active and latent) included individuals with normal medical examinations who were not suffering from acute and chronic diseases. Controls were selected from relatives of the patients with tuberculosis who had negative PPD test result. The 2 groups were matched by sex and age. The CRP levels were checked in all samples to rule out inflammatory diseases in the control group and acute phase of TB, because MBL increases in the acute phase of inflammation.

Informed consent from was obtained from all those surveyed. Then, $10 \mathrm{~mL}$ of venous blood was collected in tubes containing EDTA anticoagulant and kept in - 80 degrees. Finally, the MBL levels were measured by ELISA kit (Human MBL HK323, Hycultbiotech Co, Netherlands) and read with the ELISA Reader at $450 \mathrm{~nm}$.-Serum levels less than $10 \mathrm{ng} / \mathrm{mL}$ were considered as deficient levels. The response to treatment after 6 months of standard treatment was classified in response to treatment and treatment failure. SPSS Version 17 was used for analysis. To compare the MBL numerical values in the 2 groups, Mann-Whitney test was performed. We applied Fisher's exact test to compare the values of classified MBL in patients and controls.

T-test and Fisher's exact test were performed to compare age and sex of the 2 groups, respectively. Logistic regression was used to assess the effect of multiple variables. In all tests, the p-value of less than 0.05 was considered statistically significant.

\section{Results}

This study was done on 62 patients with TB as the case group and 63 noninfected patients as the control group; 23 patients $(36.5 \%)$ in the control group, and $26(41.9 \%)$ in case group were male $(\mathrm{p}=0.585)$. The mean $\pm \mathrm{SD}$ age of the patients and controls was $12.5 \pm 42.7$ and $16.3 \pm 46.9$ years, respectively $(p=0.107)$. The CRP level in all participants in both groups was negative. Distribution of serum MBL levels (ng/mL) of the participants are presented in Table 1. As illustrated in Table 2, elevated MBL levels in patients with pulmonary tuberculosis were significantly higher than those of the control group, but the frequency of low (less than $10 \mathrm{ng} / \mathrm{mL}$ ), normal $(11-5000 \mathrm{ng} / \mathrm{mL}$ ), and high $(>5000 \mathrm{ng} / \mathrm{mL}) \mathrm{MBL}$ levels was not significantly different between the 2 groups.

The logistic regression was used to evaluate the effect of dependent variables such as age and gender on MBL level and no relationship was found; the results of the evaluations are demonstrated in Table 3.

There was no significant difference between the level of MBL $(\mathrm{ng} / \mathrm{mL})$ in patients with response to antituberculosis treatment (median= 1012) and patients with treatment failure $($ median $=798.9)(p=0.84)$.

\section{Discussion}

This study aimed at determining the levels of MBL in patients with pulmonary tuberculosis compared to a control group. The results revealed that decreased levels of MBL play a protective role against tuberculosis. In a study by Alagarasu et al., conducted in India in 2007, they found

Table 1. MBL Level in Patients with Tuberculosis and the Control Group

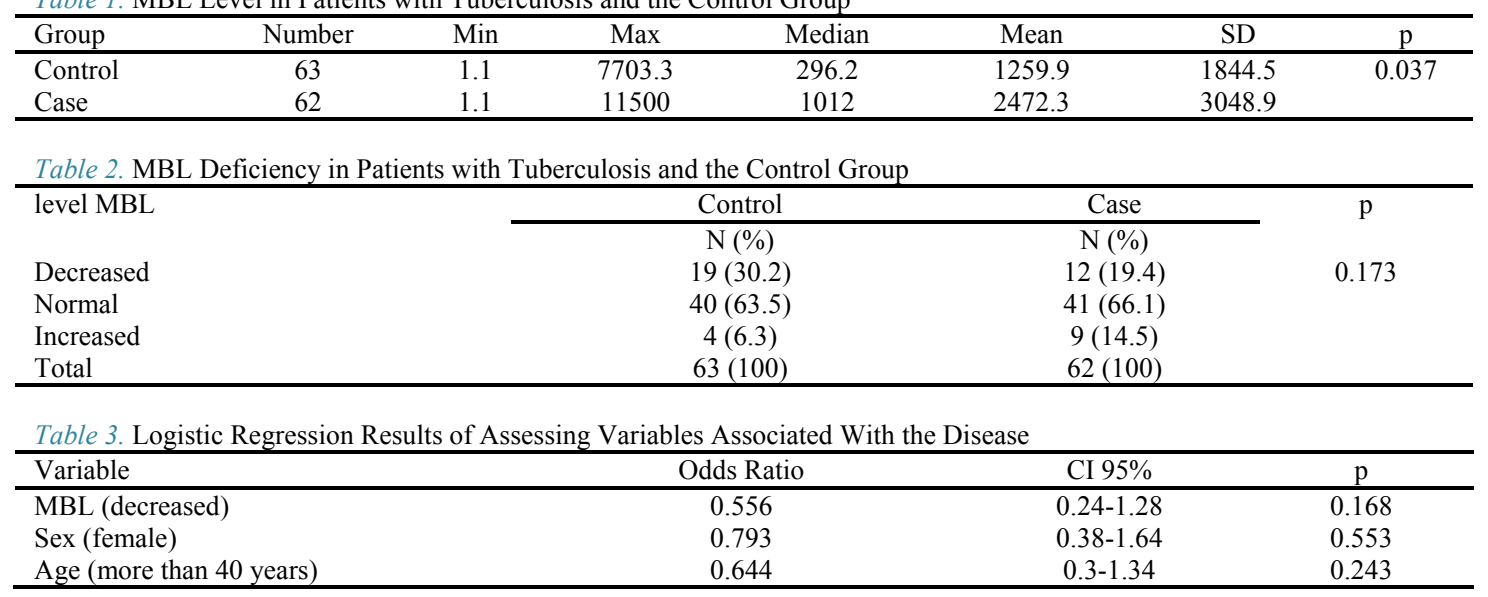


that MBL levels increased significantly among HIV-TB+ and $\mathrm{HIV}+\mathrm{TB}+$ patients compared to controls and HIV+TB- patients (16). In another study in India, Selvaraj et al. evaluated 48 patients with pulmonary tuberculosis and 58 healthy controls for MBL levels. They found significantly higher MBL levels in patients with TB than in controls (17). Also, in another study by Kosar et al. in Turkey on 44 children with TB and 99 noninfected children, the MBL levels in children with TB was observed to be significantly higher than patients without TB (18). Bonar et al. conducted a study on 68 patients with pulmonary tuberculosis, 30 patients with other lung diseases, and 64 healthy individuals as a control group. They indicated significantly higher MBL levels in TB patients than in controls and patients with lung diseases (except TB) (19). Comparison of these studies suggests that reduced levels of MBL in patients with tuberculosis, as an intracellular pathogen, may play a protective role and the entry of the pathogen inpatients with increased levels of MBL makes it more susceptible to TB. This change and increase may be due to the genetic polymorphisms in individuals.

In some studies, MBL gene polymorphisms causing a change in the protein produced by this gene are associated with a significant incidence of the disease. Thye et al. reported a significant protective association between TB and G57E variant MBL gene polymorphism (17). In another study,Capparelli et al. found that MBL can protect or predispose the host to tuberculosis, depending on the host's haplotype pair (20).

However, in this study, serum levels of MBL in patients were observed to be significantly higher than those of the control group. Reduced amounts of serum levels lower than $10 \mathrm{ng} / \mathrm{mL}$, which showed no significant difference between the 2 groups, might have been due to the small sample size. In a study conducted by Bonar et al. in Poland, it was found that the MBL levels reduced both in patients with TB and healthy participants (19), which is similar to our results. A study was performed in 1994 by Garred et al., in which 173 patients with TB and HIV were compared with 94 TB patients who were not HIV positive and 113 controls. MBL deficiency was observed among $12.1 \%$ of the patients with TB and HIV, and $3.5 \%$ of the controls, with a significant difference. However, no significant difference was found between the control group and another group that only had tuberculosis (21). Hoal-Van Helden et al. in 1999 examined 91 patients with pulmonary tuberculosis, 64 patients with tuberculous meningitis, and 79 non-TB patients. They observed significantly higher rate of MBL deficiency in the control group (28\%) compared to that of the patients with pulmonary tuberculosis $(13 \%)$, or tuberculous meningitis (8\%) (22).

No significant difference was detected in MBL level and response to antituberculosis treatment. No similar study was found about MBL level and response to antituberculosis treatment. However, Esmat et al. reported no association between serum MBL concentration and progression of $\mathrm{HCV}$ - related liver disease in Egyptian patients with chronic hepatitis C. However, they found that responders to INF-based therapy had significantly higher serum MBL than nonresponders (23). Further stud- ies are needed to evaluate the role of $\mathrm{MBL}$ in response to antituberculosis therapy in these patients.

\section{Conclusion}

The results of the present study revealed that MBL may be involved in the pathogenesis of tuberculosis and in the low values that are protective against tuberculosis. Moreover, it seems that it has no effect on antituberculosis treatment response.

\section{Acknowledgements}

This study was supported and approved by Ahwaz Jundishapur University of Medical Sciences.

\section{Conflict of Interests}

The authors declare that they have no competing interests.

\section{References}

1. Chu SF, Tam CM, Wong HS, Kam KM, Lau YL, Chiang AK. Association between RANTES functional polymorphisms and tuberculosis in Hong Kong Chinese. Genes Immun. 2007;8(6):475-9.

2. Selvaraj P, Alagarasu K, Singh B, Afsal K. CCL5 (RANTES) gene polymorphisms in pulmonary tuberculosis patients of south India. Int J Immunogenet. 2011; 38(5):397-402.

3. Berrington WR, Hawn TR. Mycobacterium tuberculosis, macrophages, and the innate immune response: does common variation matter? Immunol Rev. 2007; 219:167-86.

4. Tso HW, Lau YL, Tam CM, Wong HS, Chiang AK. Associations between IL12B polymorphisms and tuberculosis in the Hong Kong Chinese population. J Infect Dis. 2004; 190(5): 913-919.

5. Tso HW, Ip WK, Chong WP, Tam CM, Chiang AK, Lau YL. Association of interferon gamma and interleukin 10 genes with tuberculosis in Hong Kong Chinese. Genes Immun. 2005; 6(4):358-363.

6. Jamieson SE, Miller EN, Black GF, Peacock CS, Cordell HJ, Howson $\mathrm{JM}$ et al. Evidence for a cluster of genes on chromosome 17q11-q21 controlling susceptibility to tuberculosis and leprosy in Brazilians. Genes Immun. 2004; 5(1): 46-57.

7. Bellamy R, Ruwende C, Corrah T, McAdam KP, Whittle HC, Hill $A V$. Variations in the NRAMP1 gene and susceptibility to tuberculosis in West Africans. N Engl J Med. 1998; 338(10):640-4.

8. Nauta AJ, Daha MR, van Kooten C, Roos A. Recognition and clearance of apoptotic cells: a role for complement and pentraxins. Trends Immunol. 2003; 24: 148-154.

9. Turner MW. The role of mannose-binding lectin in health and disease. Mol Immunol. 2003; 40: 423-429.

10. Bao L, Haas M, Boackle SA, Kraus DM, Cunningham PN, Park P, et al. Transgenic expression of a soluble complement inhibitor protects against renal disease and promotes survival in MRL/lpr mice. J Immunol. 2002; 168: 3601-7.

11. Polotsky VY, Belisle JT, Mikusova K, Ezekowitz RA, Joiner KA. Interaction of human mannose-binding protein with Mycobacterium avium. J Infect Dis. 1997: 175: 1159-68.

12. Hamvas RM, Johnson M, Vlieger AM, Ling C, Sherriff A, Wade A, et al. Role for mannose binding lectin in the prevention of Mycoplasma infection. Infect Immun. 2005: 73: 5238-40.

13. Ji X, Gewurz H, Spear GT. Mannose binding lectin (MBL) and HIV Mol Immunol. 2005: 42: 145-52.

14. Garred P, Harboe M, Oettinger T, Koch C, Svejaard A. Dual role of mannan-binding protein in infections: another cases of heterotosis? Eur J Immunogenet. 1994; 21:125-31.

15. Denholm JT, McBryde ES, Eisen DP. Mannose-binding lectin and susceptibility to tuberculosis: a meta-analysis. Clin Exp Immunol. 2010; 162(1):84-90.

16. Alagarasu K, Selvaraj P, Swaminathan S, Raghavan S, Narendran G, Narayanan PR. Mannose binding lectin gene variants and susceptibility to tuberculosis in HIV-1 infected patients of South India. Tuberculosis (Edinb). 2007; 87(6):535-43.

17. Thye T, Niemann S, Walter K, Homolka S, Intemann CD, Chinbuah 
MA, et al. Variant G57E of mannose binding lectin associated with protection against tuberculosis caused by Mycobacterium africanum but not by M. tuberculosis. PLoS One. 2011; 6(6):e20908.

18. Cosar H, Ozkinay F, Onay H, Bayram N, Bakiler AR, Anil M, et al. Low levels of mannose-binding lectin confers protection against tuberculosis in Turkish children. Eur J Clin Microbiol Infect Dis. 2008; 27(12):1165-9.

19. Bonar A, Chmiela M, Rózalska B. Level of mannose-binding lectin (MBL) in patients with tuberculosis. Pneumonol Alergol Pol. 2004;72(5-6):201-5.

20. Capparelli R, Iannaccone M, Palumbo D, Medaglia C, Moscariello E, Russo A, et al. Role played by human mannose-binding lectin polymorphisms in pulmonary tuberculosis. J Infect Dis. 2009; 199(5):666-72.

21. Garred P, Richter C, Andersen AB, Madsen HO, Mtoni I, Svejgaard A, Shao J. Mannan-binding lectin in the sub-Saharan HIV and tuberculosis epidemics. Scand J Immunol. 1997; 46(2):204-8.

22. Hoal-Van Helden EG, Epstein J, Victor TC, Hon D, Lewis LA, Beyers N, Zurakowski D, Ezekowitz AB, Van Helden PD. Mannosebinding protein $\mathrm{B}$ allele confers protection against tuberculous meningitis. Pediatr Res. 1999;45(4 Pt 1):459-64.

23. Esmat S, Omran D, Sleem GA, Rashed L. Serum mannan-binding lectin in egyptian patients with chronic hepatitis $\mathrm{C}$ : its relation to disease progression and response to treatment. Hepat Mon. 2012; 12(4):259-64. 